\title{
COHOMOLOGICAL DETECTION AND REGULAR ELEMENTS IN GROUP COHOMOLOGY
}

\author{
JON F. CARLSON AND HANS-WERNER HENN
}

(Communicated by Eric Friedlander)

\begin{abstract}
Suppose that $G$ is a compact Lie group or a discrete group of finite virtual cohomological dimension and that $k$ is a field of characteristic $p>0$. Suppose that $\mathcal{O}$ is a set of elementary abelian $p$-subgroups $G$ such that the cohomology $H^{*}(B G, k)$ is detected on the centralizers of the elements of $\mathcal{O}$. Assume also that $\mathcal{O}$ is closed under conjugation and that $E$ is in $\mathcal{O}$ whenever some subgroup of $E$ is in $\mathcal{O}$. Then there exists a regular element $\zeta$ in the cohomology ring $H^{*}(B G, k)$ such that the restriction of $\zeta$ to an elementary abelian $p$-subgroup $E$ is not nilpotent if and only if $E$ is in $\mathcal{O}$. The converse of the result is a theorem of Lannes, Schwartz and the second author. The results have several implications for the depth and associated primes of the cohomology rings.
\end{abstract}

The last few years have witnessed a great deal of research on the algebraic structure of the cohomology rings of groups. The reason is that many of the ringtheoretic considerations such as varieties, associated primes and depth reflect properties of the groups and also of the topology of their classifying spaces. The evidence for such relationships has been building ever since Quillen first proved [Q] that the minimal primes of the mod- $p$ cohomology were the radicals of the kernels of restrictions to maximal elementary abelian $p$-subgroups. More generally, any associated prime of the cohomology ring is invariant under the Steenrod reduced power operations and hence must be the radical of the kernel of restriction to some elementary abelian $p$-subgroup of the group.

Recently, particularly through Lannes' work $[\mathrm{L}]$, it has become evident that the elementary abelian $p$-groups play a central role in the theory of unstable modules over the Steenrod algebra. The new developments in this theory were used in [HLS] to prove that the existence of regular elements (non-divisors of zero) in cohomology implies a detectability of mod- $p$ cohomology on the centralizers of certain elementary abelian $p$-subgroups determined by the regular element. The main purpose of this note is to strengthen that theorem (Theorem 1, below) by proving that it has a strong converse, that the detectability condition requires the existence of certain homogeneous regular elements and hence that the set of homogeneous regular elements is characterized by the detectability condition.

Received by the editors December 22, 1993.

1991 Mathematics Subject Classification. Primary 20J05, 20J06, 55R40.

The first author was partially supported by a grant from NSF.

The second author was supported by a Heisenberg grant from DFG. 
Let $p$ be a fixed prime and let $G$ be either a compact Lie group (which may be finite) or a discrete group of finite virtual cohomological dimension such that the mod- $p$ cohomology $H^{*} B G=H^{*}(B G, \mathbb{Z} / p)$ of its classifying space $B G$ is noetherian. The set of elementary abelian $p$-subgroups of $G$ will be denoted by $\mathcal{A}(G)$. For a subgroup $H$ of $G$ let $C_{G}(H)$ be its centralizer in $G$. The starting point of this paper is the result mentioned above [HLS, Corollary I.5.7].

Theorem 1. Let $G$ be as above. Assume $x \in H^{*} B G$ is not a zero divisor. Denote by $\mathrm{D}(x)$ the set of elementary abelian p-subgroups such that the restriction of $x$ to $H^{*} B E$ is not nilpotent. Then the map

$$
\prod_{E \in \mathrm{D}(x)} \operatorname{res}_{G, C_{G}(E)}: H^{*} B G \longrightarrow \prod_{E \in \mathrm{D}(x)} H^{*} B C_{G}(E)
$$

whose components are the restriction homomorphisms, is a monomorphism.

It is obvious that $\mathrm{D}(x)$ is a subset $\mathcal{O}$ of $\mathcal{A}(G)$ which has the following two properties:

i) If $E$ is in $\mathcal{O}$, then $g E g^{-1}$ is in $\mathcal{O}$ for all $g \in G$.

ii) If $E$ is in $\mathcal{O}$ and $E \subset E^{\prime}$, then $E^{\prime}$ is in $\mathcal{O}$.

Definition. A subset $\mathcal{O}$ of $\mathcal{A}(G)$ is called open if and only if $\mathcal{O}$ satisfies i) and ii) above.

In particular, $\mathrm{D}(x)$ is open for each $x \in H^{*} B G$. We remark that there is a topology on the set $\mathcal{A}(G)$ whose open subsets are precisely those which are open in the sense of the definition. In fact, conjugacy classes of elementary abelian $p$-subgroups correspond to prime ideals which are invariant under the Steenrod power operations $[\mathrm{Q}]$, and via this correspondence the open sets in this topology correspond to open sets in the Zariski topology on $H^{*}(G, k)$ restricted to the set of invariant prime ideals.

In this note we investigate consequences of the injectivity of the maps

$$
\prod_{E \in \mathcal{O}} \operatorname{res}_{G, C_{G}(E)}: H^{*} B G \longrightarrow \prod_{E \in \mathcal{O}} H^{*} B C_{G}(E)
$$

where $\mathcal{O}$ is any open subset of $\mathcal{A}(G)$. Our main result combined with Theorem 1 above can be stated as follows.

Theorem 2. Let $\mathcal{O}$ be an open subset of $\mathcal{A}(G)$. Then the following statements are equivalent:

(a) $\prod_{E \in \mathcal{O}} \operatorname{res}_{G, C_{G}(E)}: H^{*} B G \rightarrow \prod_{E \in \mathcal{O}} H^{*} B C_{G}(E)$ is injective.

(b) There exists $x \in H^{*} B G$ such that $\mathrm{D}(x)=\mathcal{O}$ and $x$ is not a divisor of zero.

(c) If $x$ is any element in $H^{*} B G$ with $\mathrm{D}(x)=\mathcal{O}$, then $x$ is not a divisor of zero.

Of course, the implication (b) $\Rightarrow$ (a) is just Theorem 1 , so we only have to prove that $(\mathrm{a}) \Rightarrow(\mathrm{c})$ and $(\mathrm{c}) \Rightarrow(\mathrm{b})$. As a by-product of our proof we will also get restrictions for prime ideals of $H^{*} B G$ to be associated prime ideals.

The implication $(\mathrm{c}) \Rightarrow(\mathrm{b})$ of Theorem 2 is a consequence of the following. 
Proposition 3. Let $\mathcal{O} \subset \mathcal{A}(G)$ be an open subset. Then there is an element $x \in H^{*} B G$ with $\mathrm{D}(x)=\mathcal{O}$.

Proof. The argument that follows is very standard, and occurs several places in the literature of the area. The first paragraph can be derived from (11.4) of [Q], though we don't really need anything as strong as that result. By [Q], (6.3), there are only finitely many conjugacy classes of elementary abelian $p$-subgroups of $G$. Let $E_{1}, \ldots, E_{t}$ be a complete set of representatives of the conjugacy classes of minimal elements of $\mathcal{O}$.

First we show that there exist elements $\xi_{i} \in H^{*} G$ such that $\mathrm{D}\left(\xi_{i}\right)$ consists precisely of those $E$ in $\mathcal{A}(G)$ such that $E_{i}$ is conjugate to a subgroup of $E$. For this we consider $\mathcal{C}=\{E \in \mathcal{A}(G) \mid E \notin \mathcal{O}\}$ and let $C_{1}, \ldots, C_{s}$ be representatives of the conjugacy classes of maximal elements of $\mathcal{C}$. Notice that $E_{i} \not \subseteq g E_{j} g^{-1}$ for any $j \neq i$ and $g \in G$. It is a consequence of (11.2) of [Q] that the radicals of the kernels of the restrictions to nonconjugate elementary abelian $p$-subgroups define distinct prime ideals in $H^{*}(G, k)$. Hence the radical of the kernel of restriction to $g E_{j} g^{-1}$ is not contained in the radical of the kernel of restriction to $E_{i}$ and therefore there exists an element $\gamma_{i j} \in H^{*}(G, k)$ which is homogeneous and which has the property that $\operatorname{res}_{G, E_{i}}\left(\gamma_{i j}\right)$ is not a divisor of zero while $\operatorname{res}_{G, E_{j}}\left(\gamma_{i j}\right)=0$. Likewise $E_{i} \not \nsubseteq g C_{j} g^{-1}$ for any $j$ and any $g \in G$. So for $j=1, \ldots, s$ there is a homogeneous element $\gamma_{i j}^{\prime} \in H^{*}(G, k)$ such that $\operatorname{res}_{G, E_{i}}\left(\gamma_{i j}^{\prime}\right)$ is a regular element but $\operatorname{res}_{G, C_{j}}\left(\gamma_{i j}^{\prime}\right)=0$. Let $\xi_{i}=\prod_{j=1}^{s} \gamma_{i j}^{\prime} \cdot \prod_{i \neq j} \gamma_{i j}$. Then $\mathrm{D}\left(\xi_{i}\right)$ consists precisely of those $E$ in $\mathcal{A}(G)$ such that $E_{i}$ is conjugate to a subgroup of $E$.

Now we may assume that all $\xi_{i}$ are of the same degree, by replacing each $\xi_{i}$ by some power of itself. Then consider $\xi=\sum_{i=1}^{t} \xi_{i}$. Because the $E_{i}$ 's run through a complete set of representatives of conjugacy classes of minimal elements of $\mathcal{O}$, we find that

$$
\mathrm{D}(\xi)=\bigcup \mathrm{D}\left(\xi_{i}\right)=\mathcal{O}
$$

The implication $(a) \Rightarrow(c)$ is a straightforward consequence of the fact that if $x$ is an element with $\mathrm{D}(x)=\mathcal{O}$, then by the following proposition, $\operatorname{res}_{G, C_{G}(E)}(x)$ is not a divisor of zero. Hence this will complete the proof of Theorem 2 .

Proposition 4 (cf. $[\mathrm{BH}])$. Assume $y \in H^{*} B G$ is an element whose restriction to some non-trivial central subgroup $C$ is not nilpotent. Then $y$ is not a zero divisor.

Proof. Take any non-trivial $z \in H^{*} B G$ and assume $y z=0$. Consider the homomorphism $\mu: C \times G \rightarrow G$, induced by multiplication in $G$. Then we get $0=\mu^{*}(y z)=$ $\mu^{*}(y) \cdot \mu^{*}(z)$. Now we write $\mu^{*}(y)=\operatorname{res}_{G, C}(y) \otimes 1+\tilde{y}$ with $\tilde{y} \in H^{*} B C \otimes \widetilde{H}^{*} B G$, and $\mu^{*}(z)=\Sigma z_{i}$ with $z_{i} \in H^{i} B C \otimes H^{*} B G$. Then $z_{0}=1 \otimes z$ is non-trivial and so there is a maximal $i_{0}$ such that $z_{i_{0}} \neq 0$. Then $\mu^{*}(y z)=\left(\operatorname{res}_{G, C}(y) \otimes 1\right) \cdot z_{i_{0}}+w$ with $w \in \bigoplus_{i<|y|+i_{0}} H^{i} B C \otimes H^{*} B G$. Consequently we get $\left(\operatorname{res}_{G, C}(y) \otimes 1\right) \cdot z_{i_{0}}=0$ and hence $\operatorname{res}_{G, C}(y)$ is a divisor of zero in $H^{*} B C$. Because $C$ is finite abelian, this is equivalent to saying that $\operatorname{res}_{G, C}(y)$ is nilpotent, in contradiction to our assumption.

Now we turn to associated primes in $H^{*} B G$. First we recall from [D2] or [LS] that associated primes of $H^{*} B G$ are invariant with respect to the actions of the 
Steenrod reduced power operations $P^{i}$. By (12.1) of [Q], such invariant prime ideals $\wp$ are of the following form: There exists an elementary abelian $p$-subgroup $E$ of $G$ such that $\wp=\operatorname{res}_{G, E}^{-1}(\sqrt{0})$.

The following result is here presented as a consequence of Proposition 3 and Proposition 4. It could have also been deduced from the results of Duflot in [D1, D2].

Proposition 5. Assume $\wp=\operatorname{res}_{G, E}^{-1}(\sqrt{0})$ is an associated prime. Then $E$ contains all central elements of $G$ of order $p$.

Proof. For $x \in G$ central of order $p$ consider $\langle x\rangle$, the subgroup generated by $x$. Then $\{D \in \mathcal{A}(G) \mid\langle x\rangle \subset D\}$ is open. Assume $\langle x\rangle \not \subset E$. By Proposition 3 we find $\xi \in H^{*} B G$ such that $\operatorname{res}_{G,\langle x\rangle} \xi$ is not nilpotent and $\operatorname{res}_{G, E} \xi=0$, i.e. $\xi \in \wp$. By Proposition 4 we find that $\xi$ is not a zero divisor, i.e. $\xi \notin \wp$, and we have arrived at a contradiction.

Remarks 6 . We end the paper by pointing out a few of the easier applications of Theorems 1 and 2 .

i) It is not difficult to see that if the cohomology $\operatorname{ring} H^{*} B G$ is Cohen-Macaulay, then all of the cohomology must be detected on the centralizers of the maximal elementary abelian $p$-subgroups, and moreover that the maximal elementary abelian $p$-subgroups must all have the same rank $r$. The last fact follows from a general theorem that the components of $V_{G}$ must have the same dimension. However we can derive a proof of the theorem in the case of our cohomology rings from the results of this paper. For suppose that $V_{G}$ is the maximal ideal spectrum of $H^{*} B G$, and let $V_{G}(\zeta)$ denote the set of all maximal ideals containing an element $\zeta \in H^{*} B G$. By Proposition 3, it is possible to find a homogeneous element $\zeta$ such that for every elementary abelian $p$-group $E, \operatorname{res}_{G, E}(\zeta)$ is not zero and is not nilpotent if and only if $E$ has maximal rank $r$. So $\operatorname{dim} V_{G}(\zeta)=r-1$ and we can find $\zeta_{2}, \ldots, \zeta_{r}$ such that $V_{G}(\zeta) \cap V_{G}\left(\zeta_{2}\right) \cap \cdots \cap V_{G}\left(\zeta_{r}\right)=0$. That is, each $\zeta_{i}$ is chosen so that $\operatorname{dim}\left(V_{G}(\zeta) \cap \cdots \cap V_{G}\left(\zeta_{i}\right)\right)=r-i$, or the dimension decreases by one with each additional intersection. Then $\zeta, \zeta_{2}, \ldots, \zeta_{r}$ is a system of parameters for $H^{*} B G$ and hence, by the Cohen-Macaulay assumption, is a regular sequence (see Theorem 17.4 of $[\mathrm{M}])$. In particular, $\zeta$ is regular and $\mathrm{D}(\zeta)$ is precisely the set of elementary abelian $p$-subgroups of rank $r$. Now if $\mathrm{G}$ has a maximal elementary abelian $p$-subgroup of rank less than $r$ or, equivalently, if $V_{G}$ has a component of dimension less than $r$, then we get a contradiction to the assumption that $\zeta$ is a not a divisor of zero. The reason is that then there would necessarily be an element $\gamma$ whose restriction to the particular elementary abelian $p$-subgroup would not be nilpotent but whose restriction to any rank $r$ subgroups would vanish. Thus $\zeta \gamma$ would be nilpotent and some power of $\zeta$ would annihilate some power of $\gamma$.

ii) The result of (i) above can also be shown using the methods involving transfer maps as in $[\mathrm{C}]$. For assuming that $H^{*} B G$ is Cohen-Macaulay, we may choose a set of parameters in which one of them is a sum of transfers from the centralizers of the elementary abelian $p$-subgroups of maximal rank $r$ ([C], Proposition 2.4). By Frobenius reciprocity, this element would annihilate any element whose restriction to the centralizer of every elementary abelian $p$-subgroup of rank $r$ was zero.

As in $[\mathrm{C}]$ it is tempting to ask if the converse of the statement in (i) is correct. Or more generally, if the cohomology $H^{*} B G$ is detected on the centralizers of the elementary abelian $p$-subgroups of rank $t$, is it necessary that the depth of $H^{*} B G$ 
be at least $t$ ? It is not difficult to show that this question is equivalent to Question 3.1 of $[\mathrm{C}]$.

iii) Still another proof of the statement of (i) can be given by using Corollary I.5.5 of [HLS]. We do not reproduce the statement of Corollary I.5.5 here, but would like to point out that it can be considered as a technical sharpening of Corollary I.5.7 of [HLS], i.e. of Theorem 1 of this paper.

iv) Theorems 1 and 2 can be used to prove the detectability of cohomology in certain circumstances. An example is given in (II, 5.4.2) of [HLS]. There the mod-2 cohomology of the group

$$
G=G L(2, q), \quad q \equiv 3 \quad(\bmod 4)
$$

is considered. In this case each maximal elementary abelian 2-subgroup of $G$ is conjugate to the subgroup $E$ of diagonal matrices of order 2. By exhibiting an element $x$ of $H^{*} B G$ which is not a divisor of zero and such that $\mathrm{D}(x)$ consists precisely of the conjugates of $E$, one sees via Theorem 1 that the restriction map from $H^{*} B G$ to $H^{*} B C_{G}(E)$ is injective.

The Sylow 2-subgroup of $G$ is a semi-dihedral group whose mod-2 cohomology has depth one while the cohomology of $G$ has depth 2. Other examples of this phenomenon are the mod-2 cohomology of the Mathieu group $M_{11}[\mathrm{BC}]$, and $S L(3, q)$, $q$ congruent to 3 modulo 4 (see $[\mathrm{MP}]$ ).

v) In Section I.5 of [HLS] there is another application of these ideas to infinite groups. Dwyer (private communication) has shown that for some $n$, the mod-2 cohomology of $B G L\left(n, \mathbb{Z}\left[\frac{1}{2}\right]\right)$ is not detected on the centralizer of the subgroup $E$ of all diagonal matrices of order 2. It is not difficult to exhibit an element $x$ in $H^{*} B G L\left(n, \mathbb{Z}\left[\frac{1}{2}\right]\right)$ which is the restriction of an element in $H^{*} B G L(n, \mathbb{C})$ and such that $\mathrm{D}(x)$ is again precisely the conjugates of $E$. By Theorem 1 this element must be a zero divisor and thus it gives an example which is contrary to a conjecture of Quillen $([\mathrm{Q}]$, page 591$)$ in that $H^{*} B G L\left(n, \mathbb{Z}\left[\frac{1}{2}\right]\right)$ can not be a free module over $H^{*} B G L(n, \mathbb{C})$.

\section{REFERENCES}

[BC] D. J. Benson and J. F. Carlson, Diagrammatic methods for group representations and cohomology, Comm. in Algebra 15 (1987), 53-121. MR 87m:20032

[BH] C. Broto and H.-W. Henn, Some remarks on central elementary abelian p-subgroups and cohomology of classifying spaces, Quarterly Journal of Mathematics 44 (1993), 155-163. MR 94c: 57060

[C] J. F. Carlson, Depth and transfer maps in the cohomology of groups, Math Zeit. 218 (1995), 461-468.

[D1] J. Duflot, Depth and equivariant cohomology, Comment. Math. Helv. 56 (1981), 627-637. MR 83g:57029

[D2] ㄴ. The associated primes of $H_{G}^{*} X$, J. Pure Appl. Algebra 30 (1983), 131-141. MR 85b:57040

[L] J. Lannes, Sur les espaces fonctionnels dont la source est le classificant d'un p-groupe abélien élémentaire, Publ. Math. IHES 75 (1992), 135-244. MR 93j:55019

[HLS] H.-W. Henn, J. Lannes and L. Schwartz, Localizations of unstable A-modules and equivariant $\bmod p$ cohomology, Math. Ann. 301 (1995), 23-68. CMP 95:06

[LS] P.S. Landweber and R.E. Stong, The depth of rings of invariants over finite fields, Number theory, New York, pp. 1984-1985; (Lect. Notes in Math. vol. 1240), Berlin Heidelberg New York: Springer 1987. MR 88k:13004

[M] H. Matsumura, Commutative Ring Theory, Cambridge University Press, Cambridge, 1986. 
[MP] J. Martino and S. Priddy, Classification of BG for groups with dihedral or quatenion Sylow 2-subgroups, J. Pure App. Algebra 73 (1991), 13-21. MR 92f:55022

[Q] D. Quillen, The spectrum of an equivariant cohomology ring I, II, Ann. of Math. 94 (1971), 549-602. MR 45:7743

Department of Mathematics, University of Georgia, Athens, Georgia 30602

E-mail address: jfc@sloth.math.uga.ed

Mathematisches Institut der Universität, Im Neuenheimer Feld 288, D-69120 HeidelBerg, Federal Republic of Germany

E-mail address: henn@mathi.uni-heidelberg.de 